\title{
Approaches to research utilization and capacity strengthening
}

Project SOAR

Follow this and additional works at: https://knowledgecommons.popcouncil.org/departments_sbsr-hiv

Part of the Health Services Research Commons

How does access to this work benefit you? Let us know!

\section{Recommended Citation}

Project SOAR. 2021. "Approaches to research utilization and capacity strengthening," Learnings from Project SOAR, Synthesis Brief. Washington, DC: Population Council. 


\section{Approaches to Research Utilization and Capacity Strengthening}

\section{Learnings from Project SOAR}

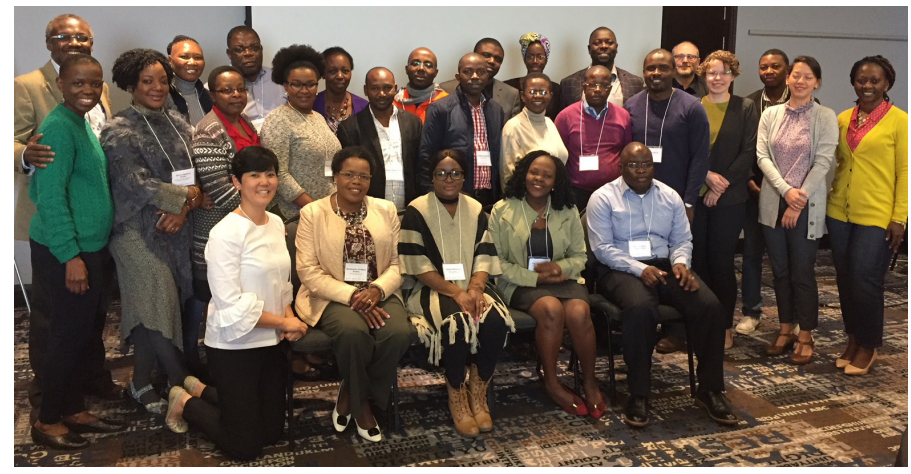

It is well established that research can play a critical role in strengthening programming and policy making. Donors and other stakeholders are increasingly focused on ensuring that their research investments yield positive health and development outcomes. Yet, there are many challenges in ensuring study findings are used by relevant decisionmakers to inform policies and programs. ${ }^{1}$

Recognizing the need to invest in research uptake efforts beyond dissemination of final findings, Project SOAR utilized an active approach to facilitating research utilization (RU) to fulfil the commitment of implementation science to "promote the systematic uptake of research findings [...] into routine practice." ${ }^{2}$ SOAR assembled and implemented a systematic and proactive RU strategy to advance translation of its robust portfolio of HIV implementation research into evidence-informed practice.

This brief synthesizes key lessons learned from implementing SOAR's RU approach across 76 research activities in 21 countries, including:

- Processes to foster local engagement

- Strategies to support capacity strengthening (CS)

- Approaches to promote knowledge translation and evidence use

\section{KEY RECOMMENDATIONS}

\section{Foster Local Engagement}

1. Engage stakeholders through research advisory committees and/or technical working groups at all stages of the research process to ensure research is responsive to local program and policy landscapes.

2. Include at least one in-country coprincipal investigator to promote meaningful stakeholder collaboration throughout the research process and foster co-ownership of results.

\section{Support Capacity Strengthening}

3. Provide comprehensive tools, trainings, and mentorship to strengthen implementation science and research utilization skill sets among key stakeholders.

\section{Promote Knowledge Translation and Use}

4. Commit dedicated research utilization resources to provide technical support and knowledge translation expertise, facilitate research utilization processes, and ensure utilization activities are reflected in research plans and budgets.

5. Disseminate evidence routinely, including interim findings, in formats tailored for different audiences to inform ongoing program improvements and promote evidence use. 


\section{OVERVIEW OF SOAR'S RU PROCESS AND TOOLS}

Early in the project, SOAR developed guidance on its "Approach to Research Utilization," which describes seven key practices to foster RU throughout the operations research (OR) process and includes tools to facilitate each practice. ${ }^{3}$ SOAR's RU process begins with the identification and engagement of key stakeholders during study design to ensure research questions respond to local program and policy needs. Engagement of stakeholders through a research advisory committee (RAC) continues throughout study implementation, including data collection, analysis and interpretation. Finally, this RAC works together to disseminate results, formulate relevant recommendations, and champion evidence-informed policy and/or program change.

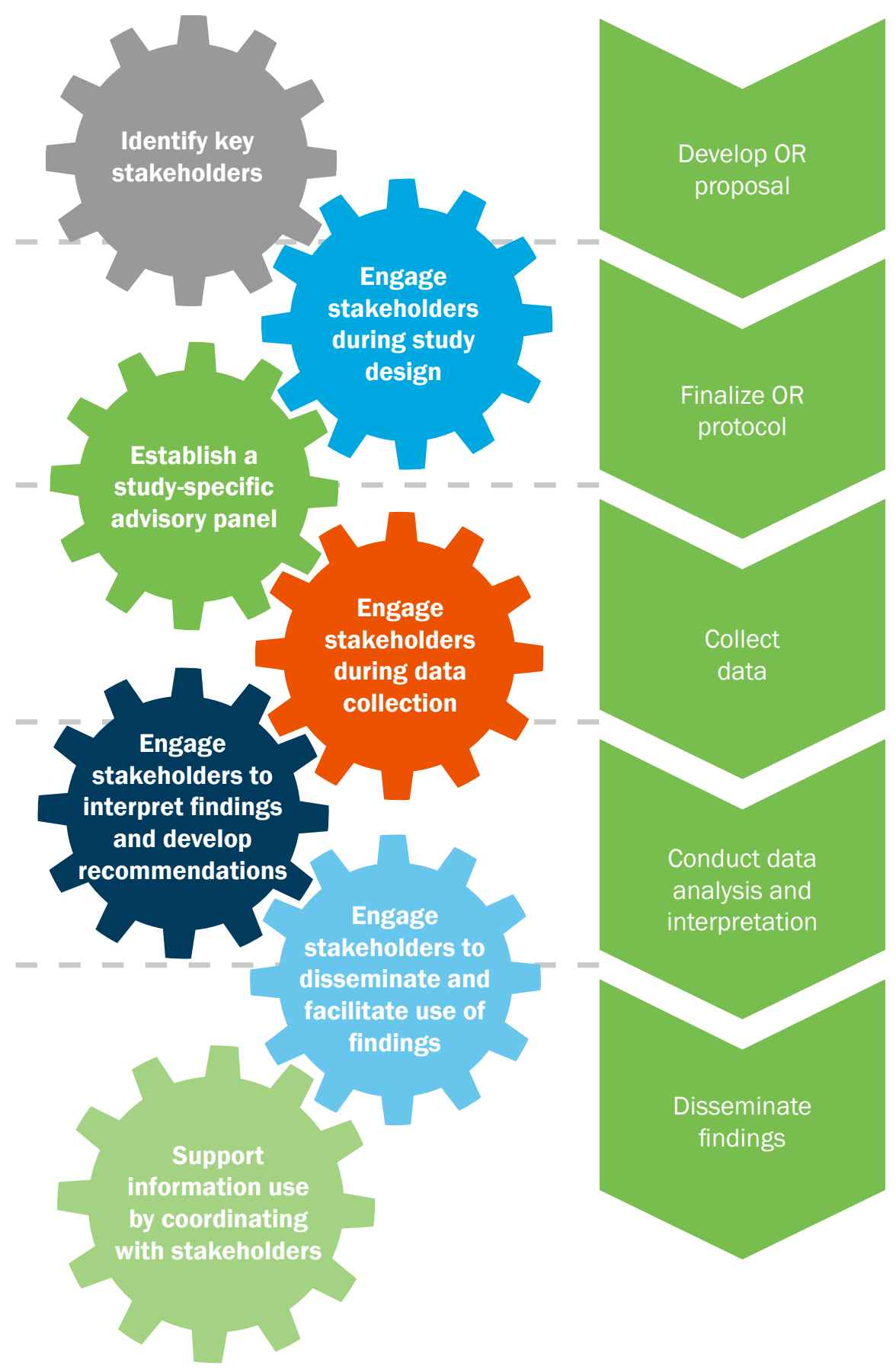

\section{ABOUT}

Project SOAR (Supporting Operational AIDS Research), was a 6-year USAID-funded implementation science initiative with over 70 activities in 21 countries. The Population Council led Project SOAR in collaboration with Avenir Health, Elizabeth Glaser Pediatric AIDS Foundation (EGPAF), Johns Hopkins University, Palladium, and The University of North Carolina at Chapel Hill.

\section{Series}

"Learnings from Project SOAR" seeks to highlight key results, recommendations, and examples of research impact across this 6-year, 21-country initiative.

The series includes five thematic briefs focused on biomedical prevention interventions, HIV testing services, HIV treatment strategies, stigma, and research utilization and capacity strengthening. 


\section{FOSTERING LOCAL ENGAGEMENT}

Summary: Identifying stakeholders who can

provide input into study development and

implementation, as well as promote translation

of findings into policy and practice, is a

foundational aspect of the RU process. SOAR's

research consistently included principal

and co-investigators from study countries to

ensure research agendas were responsive

to country needs and promote co-ownership

of results. Also, before initiating a study,

SOAR researchers assessed opportunities

to use existing national forums as platforms

for engaging stakeholders throughout the

research process. Pre-existing technical

working groups, as well as newly formed RACs,

provided critical venues for formulating study

plans, sharing interim and final results, and

creating dissemination plans.

A cornerstone of the RU process, as described in SOAR's RU Guide, is identifying stakeholders who can provide input throughout the study and promote the translation of research results into policy and practice. This knowledge translation can range from a change in the thinking about a problem, to a commitment to act, to tangible action for change. SOAR promoted the engagement of stakeholders at many points in the research process, including through RACs and/ or technical working groups (TWGs) as well as the involvement of co-principal investigators in study countries.

\section{Research advisory committees and technical working groups}

For some SOAR studies, researchers identified preexisting TWGs-usually convened by the national AIDS coordinating body-and worked with them as forums for obtaining input from stakeholders and disseminating and discussing findings. In most SOAR studies, however, it was necessary for researchers to form study-specific RACs. SOAR's Stakeholder Engagement Tool provided a structured approach with key questions to optimize the identification of stakeholders and define their respective roles.
Membership: While the composition of TWGs and RACs varied, the most common stakeholders engaged were from national AIDS coordinating bodies (e.g., the National AIDS Coordinating Council in Kenya or the National AIDS Council in Zambia), ministry of health HIV programs at national and sub-national levels, President's Emergency Plan for AIDS Relief (PEPFAR) country teams (the donor), intervention implementation partners, and community advocates. Other RAC members were specific to the focus of the study. Beyond the national HIV programs, researchers prioritized engagement of appropriate government and non-governmental sectors for the topic being studied. Some examples are illustrated in Box 1.

Core activities: Project SOAR established 54 RACs and facilitated the RACs to develop 46 in-country data use plans. The RACs and TWGs were invaluable throughout the research process, including study conceptualization and launch. For example, RAC members in Zambia proposed to investigators planning a study among HIV-positive orphans and other vulnerable children (OVC) that community-based counselors supporting the OVC be oriented to the study. This would then enable the counselors to orient OVC and their caregivers about the study before data collectors approached their homes. Box 1 Examples of country-level RAC
membership

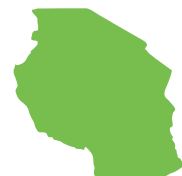

In Tanzania, a study on the family planning needs of people living with HIV engaged the reproductive health division of the Ministry of Health. Donors and service providers, who are crucial for taking up findings to improve services, also became RAC members.

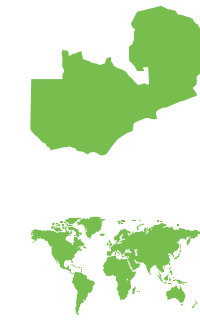

In Zambia, the RAC for the Project YES! study, which tested a peer mentor program for young people living with HIV (YPLHIV), included YPLHIV as members.

Globally, SOAR established a working group featuring representatives from the Global Network of People Living with HIV and the International Community of Women Living with HIV to guide the process. 
Also, SOAR researchers took advantage of existing forums, such as TWGs or newly established RACs, to hold periodic meetings throughout the course of the study to discuss the data and its implications. In countries with multiple SOAR studies (e.g., Malawi, South Africa, Tanzania, and Zambia), SOAR held joint RAC meetings at the national level, which helped to conserve time and resources, and facilitated participation of USAID and government representatives.

Additionally, SOAR encouraged RACs to develop written plans that described how the data would be communicated and used. RACs identified and coached champions to continue engaging stakeholders in various forums to integrate study findings into decision making processes.

\section{Co-investigators from study countries}

From the outset of the project, SOAR committed to and consistently involved co-principal investigators from study countries as core members of the research team. In total, 135 in-country researchers and other stakeholders collaborated as co-investigators across SOAR's implementation science portfolio. These co-

\section{Box 2 Why understanding stakeholder, program} and policy landscapes is critical to RU

In a commentary published in AIDS and Behavior, SOAR provided recommendations for actively promotion of research uptake using emerging lessons from the project's RU process. "The mechanism through which research influences policy and program change is not linear.

Stakeholders respond to multiple factors, not just the evidence from studies, to make decisions. Translating research findings into action thus requires assessment of the research-and-policy/ program dynamics within which the study is being conducted. In addition, lack of timeliness or opportunity to use the research is one of the most prominent barriers to RU. Hence, we recommend efforts to encourage RU seek to understand the times and opportunities for influencing policy/ program change together with contextual factors that may influence decision making." investigators were often members of local research institutions. They also included collaborators from implementing organizations as well as officials from ministries of health, national HIV/AIDS authorities, or other government agencies. For instance, a study to assess the impacts of the Namibia's 2017 rollout of its "test and treat" policy engaged co-investigators from key institutions and organizations in the country. Co-investigators affiliated with an implementing partner that supported provision of facility services in Namibia were essential to ensuring access to facility staff and secondary data. Another co-investigator with the Ministry of Health and Social Welfare also provided critical inputs to ensure the study was responsive to the Namibian government's questions and information needs.

\section{CAPACITY STRENGTHENING}

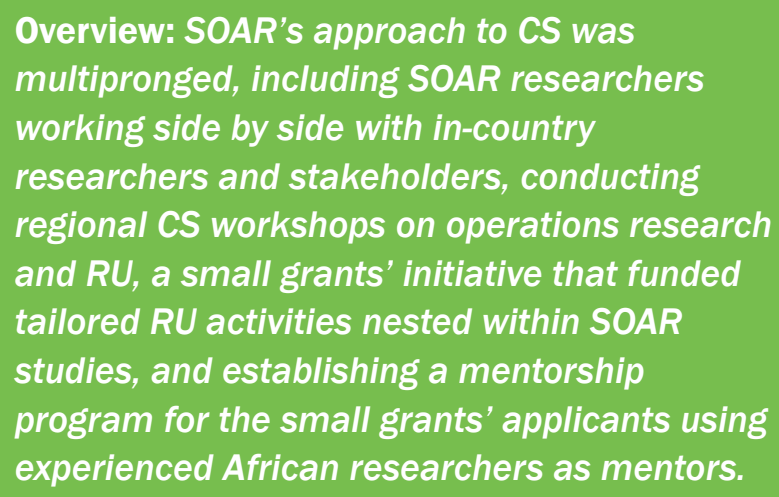

Overview: SOAR's approach to CS was

multipronged, including SOAR researchers

working side by side with in-country

researchers and stakeholders, conducting

regional CS workshops on operations research

and RU, a small grants' initiative that funded

tailored RU activities nested within SOAR

studies, and establishing a mentorship

program for the small grants' applicants using

experienced African researchers as mentors.

\section{Role of capacity strengthening workshops}

In February 2017, SOAR conducted a 4-day capacity strengthening (CS) workshop to enhance individual and institutional capacity in HIV implementation science and operations research, with an emphasis on the RU process. The 28 workshop participants were in-country researchers working on 19 SOAR studies in 12 countries, and each researcher was joined by a study stakeholder from a governmental or nongovernmental institution responsible for program or policy implementation-a key innovation of the workshop. Participants discussed the communication needs of different audiences; criteria for choosing which findings to highlight; and how to identify a key finding, communicate its implications, and provide recommendations. Participants also shared 
insights and challenges to communicate results with stakeholders and broader audiences. Notably, shortly after the workshop, two participants secured funding through a regional small grants programs based on proposals they developed at the workshop. ${ }^{4}$

At the conclusion of the workshop, SOAR introduced a small grants program designed to strengthen research utilization, knowledge translation, and secondary analysis efforts. In addition, SOAR convened a followup CS workshop in May 2018 for grantees and their mentors. This second workshop included 20 workshop participants from eight African countries and focused on strengths and weaknesses identified in the small grant applications as well as mentorship and leadership skills. Based on a follow-up survey six months after the workshop, every participant who developed goals with their mentor achieved at least one of those goals. ${ }^{5}$

\section{Small grants initiative}

SOAR implemented a competitive small grants initiative whereby researchers working on SOAR studies could receive additional funds to apply the skills they learned at the 2017 CS workshop. One grantee was the National Forum of People Living with HIV/AIDS Networks in Uganda (NAFOPHANU), who collaborated with Project SOAR in testing an updated version of the People Living with HIV (PLHIV) Stigma Index. The grant allowed NAFOPHANU to hold local stakeholder meetings to discuss the findings in the six districts where the study was conducted and the production of six district-based research briefs. It also created a community of practice linked to the Uganda AIDS Commission enabling data from the study to be used in developing the national stigma policy.

\section{KNOWLEDGE TRANSLATION \& USE}

Overview: SOAR found that using a proactive,

systematic approach to $R U$ contributed to

evidence-based program improvements and

policy changes to enhance HIV prevention,

care and treatment across multiple countries.

Integration of a dedicated RU Advisor position

within the SOAR management team provided

essential, ongoing technical support to

integrate RU practices and tools consistently

across the project's portfolio. SOAR regularly

discussed both interim and final results with

stakeholders, providing timely, relevant, and

quality evidence to improve ongoing program

performance.

\section{Critical role of dedicated $\mathrm{RU}$ resources and staff}

Ensuring that study resources are allocated to $\mathrm{RU}$ activities can be a key factor in successfully translating evidence into practice. One important way Project SOAR dedicated resources to knowledge translation and evidence use was through the creation of a dedicated RU Advisor position within its global management team. The RU Advisor provided continuous technical support to research teams from study inception through dissemination of findings, including:

- Ensuring RU steps were integrated into study protocols

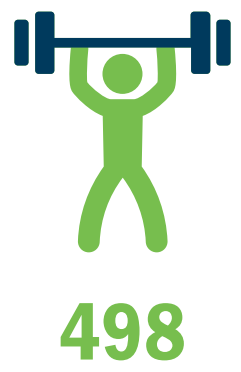

in-country researchers have participated in a SOAR capacity strengthening activity
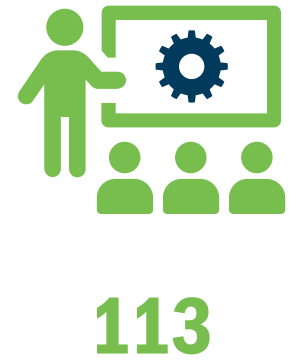

local institutions have received targeted SOAR activities designed to strengthen their research capacity

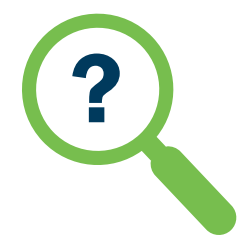

135

in-country researchers and other stakeholders serving as co-investigators on SOAR studies 
- Helping identify key stakeholders and policy issues relevant to study topics

- Contributing to preparation for and facilitation of RACs, TWGs, and other meetings with stakeholders

- Strengthening the capacity of in-country investigators and stakeholders to review and use research to improve programs/policies.

In addition, the RU Advisor played a critical role in ensuring dissemination workshops provided effective opportunities for open dialogue about the implications of study findings, formulation of evidence-based recommendations, and development of plans to inform policy and program decisions. The project management team also reviewed study proposals and budgets to ensure that SOAR investigators were committing the necessary resources for RU activities and stakeholder engagement. Both the dedicated $\mathrm{RU}$ Advisor position and commitment of financial resources for RU within study proposals and budgets were critical for catalyzing knowledge translation and use given the size, scope, and complexity of the SOAR portfolio.

\section{Importance of dissemination format and frequency}

SOAR researchers frequently shared preliminary results with stakeholders, providing the opportunity to use emerging data to adjust programs in real time. To enable stakeholders to access interim study findings, SOAR conducted data interpretation meetings. Results briefs were also produced in user-friendly formats highlighting key findings and programmatic implications for busy decisionmakers, including national government and USAID teams, which contributed to data-driven operational planning. Additionally, SOAR took advantage of regional and international conferences to reach a broader range of stakeholders and to share the project's learnings about RU (see Box 3).

\section{Data use to improve program performance}

\section{Box 3 Dissemination event-Enhancing utilization of HIV implementation science findings through the engagement of key decision-makers}

In July 2020, SOAR hosted a virtual workshop at the AIDS 2020 Conference to highlight case studies of collaboration between implementation science researchers, communities, program managers, and policymakers leading to evidence use across diverse contexts. ${ }^{7}$ One example focused on the role of community leadership in updating the PLHIV Stigma Index. As the update process was designed to be led and implemented by PLHIV, it has helped support evidence-based responses to stigma and discrimination. From influencing laws in countries such as Uganda, to developing and testing the PLHIV Resilience Scale to better capture community experiences, the PLHIV Stigma Index development process has showcased the value, and impact, of intentional and thorough community engagement through the research and dissemination process.
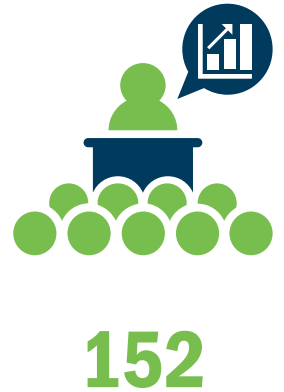

oral/poster presentations given by SOAR PIs at international, regional, and national conferences
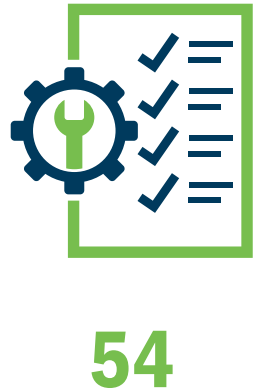

program practices, guidelines, and tools have been influenced by exposure to SOAR research
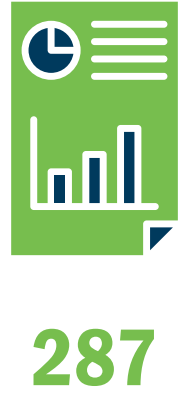

researchers and/or institutions have used SOAR's research findings 
Some stakeholders utilized SOAR study data to improve programs even before the final study findings were published. This was the result of SOAR's concerted effort to disseminate interim findings as soon as possible in a variety of formats, including inperson presentations, webinars, and research briefs, targeted to specific audiences, including PEPFAR country teams, ministries of health, and program managers.

In one South African study focused on PLHIV and tuberculosis, the research team shared contact tracing data with public clinical managers every quarter. The team reported that after reviewing firstquarter data, the clinic instituted additional contact tracing training for community health workers, which led to improved contact tracing rates the following quarter. In a second South African study on provider- initiated HIV testing and counselling, the research team shared findings identifying missed opportunities for testing with clinic staff. This led to immediate changes to close gaps in services, including offering HIV testing to clients while waiting to see a clinician, which helped to utilize time otherwise wasted. ${ }^{1}$

Baseline data from a longitudinal study in eSwatini found that 43 percent of children living with HIV were on a suboptimal antiretroviral treatment (ART) regimen (NVP-based) and those on suboptimal ART were less likely to be virally suppressed. Although not a primary research question, the study team immediately shared this finding with the Ministry of Health and worked with them to ensure suboptimal regimens were replaced. ${ }^{6}$

\section{RESEARCH IMPACT SPOTLIGHTS}

\section{Examples of evidence-informed policy and program change}

Tanzania authorized community-based ART for key populations informed by SOAR's study there demonstrating it improved ART initiation and retention. All service delivery providers were informed of this change via a formal government circular.

Senegal added HIV self-testing to its national strategy following a SOAR study showing it was feasible and acceptable among men who have sex with men.

Uganda and Eswatini used SOAR modelling results to modify its selection of priority populations to be targeted for pre-exposure prophylaxis.

Global: Following the successful revision and pilot of the PLHIV Stigma Index under SOAR in 2016-18, over 30 countries have either implemented or plan to implement the revised instrument ("PLHIV Stigma Index 2.0").

For more information, please visit "Learnings from Project SOAR" series page. 


\section{REFERENCES}

1. Kalibala, S. and T. Nutley. 2019. "Engaging stakeholders, from inception and throughout the study, is good research practice to promote use of findings," AIDS and Behavior 23(Suppl 2): 214-219. doi:10.1007/s10461-01902574-w

2. Eccles, M. P. and B. S. Mittmann BS. 2006. "Welcome to implementation science," Implementation Science 1: 1. doi: 10.1186/1748-5908-1-1

3. Project SOAR. 2016. "Project SOAR's approach to research utilization," Project SOAR. Washington, D.C.: Population Council. https://projsoar.org/wp-content/ uploads/2017/12/ResUtilization_Guidance.pdf

4. Kalbarczyk, A. et al. 2019. "Research capacity strengthening in sub-Saharan Africa: Recognizing the importance of local partnerships in designing and disseminating HIV implementation science to reach the 90-90-90 goals," AIDS and Behavior 23(Suppl 2): 206213. doi: 10.1007/s10461-019-02538-0

Suggested citation: Project SOAR. 2021. "Approaches to research utilization and capacity strengthening," Learnings from Project SOAR Synthesis Brief. Washington, D.C.: Population Council.

For more information, please visit Project SOAR.
5. Kalbarczyk, Anna, and Yukari C. Manabe. 2019. "Capacity strengthening in HIV operations research: report on second Project SOAR workshop, 15-18 May 2018, Johannesburg, South Africa," Project SOAR Final Report. Washington, DC: USAID | Project SOAR. https://projsoar. org/wp-content/uploads/2019/09/CSPhasell_Workshop_ Report.pdf

6. Chouraya, Caspian et al. 2020. "Piloting and evaluating family-centered care in Eswatini," Project SOAR Final Report. Washington, D.C.: Population Council. https:// projsoar.org/wp-content/uploads/2021/01/Eswatini_ FAMCARE_FinalReport.pdf

7. Population Council. 2020. "Enhancing utilization of HIV implementation science findings through the engagement of key decision-makers." Website commentary, 25 August. Accessed at: https://www.popcouncil.org/news/ enhancing-utilization-of-hiv-implementation-sciencefindings-through-the-en?mc_cid=e737988f35\&mc_ eid $=22244$ b $704 e$
Project SOAR was a six-year (September 2014-January 2021) cooperative agreement funded by the United States President's Emergency Plan for AIDS Relief and the U. S. Agency for International Development (Agreement No. AID-OAA-A-14-00060). The contents of this brief are the sole responsibility of Project SOAR and Population Council and do not necessarily reflect the views of PEPFAR, USAID, or the United States Government.

Population Council led the Project SOAR consortium in collaboration with Avenir Health, Elizabeth Glaser Pediatric AIDS Foundation, the Johns Hopkins University, Palladium, and The University of North Carolina at Chapel Hill.
Project SOAR/Population Council

4301 Connecticut Avenue, NW, Suite 280

Washington, DC 20008

Tel: +1 2022379400

e-mail: ProjectSOAR@popcouncil.org projsoar.org

(C) Population Council, April 2021

Cover photo credit: Project SOAR 